\title{
Group and individual academic detailing for clinicians influenced prescribing of antidepressants for the elderly
}

van Eijk ME, Avorn J, Porsius AJ, et al. Reducing prescribing of highly anticholinergic antidepressants for elderly people: randomised trial of group versus individual academic detailing. BMJ 2001 Mar 17;322:654-7.

QUESTION: In treating elderly people with depression, can academic detailing in group or individual educational visits increase prescriptions of less anticholinergic antidepressants and reduce prescriptions of highly anticholinergic antidepressants?

\section{Design}

Cluster randomised \{allocation concealed*\}t, unblinded*, controlled trial with 8 months of follow up.

\section{Setting}

The South Holland islands, southwest Netherlands.

\section{Participants}

21 peer review groups of healthcare professionals (190 general practitioners [GPs] [93\% men] and 36 pharma-

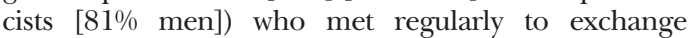
information, advise on policy, agree on guidelines, and use feedback methods to measure adherence to guidelines. Patients were $60-96$ years of age $(n=46078$, mean age 71 y, $58 \%$ women).

\section{Intervention}

Block randomisation was used to assign 3 blocks of 7 peer review groups to 1 of 3 conditions: individual educational visits $(\mathrm{n}=84$ professionals, 17143 patients), group educational visits for the peer review group as a whole ( $\mathrm{n}=61$ professionals, 12734 patients), or no visits $(\mathrm{n}=81$ professionals, 16201 patients).
Source of funding: $\mathrm{OZ}$ zorgverzekeringen (health insurance company).

For correspondence: $\operatorname{Dr} A$ de Boer, Department of

Pharmaco-epidemiology and Pharmacotherapy, Utrecht Institute of

Pharmaceutical

Sciences, Faculty of

Pharmacy, PO Box

$80082,3508 \mathrm{~TB}$

Utrecht, the

Netherlands. Fax +31

302539166.

\section{Main outcome measures}

Incident use of highly anticholinergic antidepressants and less anticholinergic antidepressants.

\section{Main results}

Results were adjusted for sex and baseline rates of incident antidepressant prescriptions. At 8 months, patients whose healthcare providers received group visits had fewer prescriptions for highly anticholinergic antidepressants than those whose healthcare providers received no visits $(\mathrm{p}=0.023)$ (table). Prescriptions for less anticholinergic antidepressants were given more often in the individual visit condition than in the no visit condition $(\mathrm{p}=0.016)$ (table). Prescription rates did not differ between the group and individual visit conditions.

Individual or group educational visits $v$ no visits for influencing incident prescription rates for elderly people with depression at 8 months

\begin{tabular}{lll} 
Antidepressant type & Comparison & $\begin{array}{l}\text { Adjusted rate ratio reduction } \\
(95 \% \mathrm{CI})\end{array}$ \\
\hline Highly anticholinergic & Individual $v$ no visits & $26 \%(-4$ to 48$) \S$ \\
\hline & Group $v$ no visits & $45 \%(8$ to 67$)$ \\
\hline & & Adjusted rate ratio increase $(\mathrm{Cl})$ \\
\hline Less anticholinergic & Individual $v$ no visits & $40 \%(6$ to 83$)$ \\
\hline & Group $v$ no visits & $29 \%(-7$ to 79$) \S$ \\
\hline
\end{tabular}

$\ddagger$ Rate ratios adjusted for sex and baseline rates of incident prescriptions. §Not statistically significant.

\section{Conclusions}

In elderly people with depression, group academic detailing for health professionals reduced prescriptions of highly anticholinergic antidepressants and individual academic detailing increased prescriptions of less anticholinergic antidepressants. Prescription rates for both types of antidepressants did not differ between group and individual visit conditions.

*See glossary. †Information provided by author.

\section{COMMENTARY}

In all age groups, newer antidepressants are used increasingly. ${ }^{1}$ Concerns have been expressed that insufficient evidence exists to support this trend in older people. ${ }^{2}$ When faced with a choice between dosage titration with an older tricyclic agent (TCA) or the simpler regimens afforded by many of the newer drugs, practitioners will often choose the latter. Furthermore, although older antidepressants are effective, they have some extremely unpleasant side effects to which the elderly in particular are prone. An intervention designed to lead to safer prescribing seems worthwhile.

The study by van Eijk et al investigates academic detailing, which is a university based educational outreach activity designed to improve practice in healthcare professionals, particularly prescribing practices. ${ }^{3}$ It is based on theories and techniques used in adult learning and marketing.

To categorise antidepressants as safer or less safe according to their anticholinergic properties risks overlooking other dimensions of safety; effects such as postural hypotension, sedation, falls, and lethality in overdose may also be found among drugs in the authors' "safer" group. For example, in the UK dothiepin (dosulepin) is still frequently prescribed but has been criticised because relatively few tablets in overdose may be lethal, while lofepramine, another tricyclic, is not listed but is widely used. ${ }^{4}$

Therefore, whatever yardstick is used to define safer or better is best left to locally agreed protocols. In the UK, this is a necessary part of implementing the National Service Framework for Older People (NSFOP). The NSFOP also charges specialist psychiatric services with providing educational support and training to primary care clinicians. Thus, it is important that new technology of this kind be examined closely by psychiatrists who treat older people. Robert C Baldwin, DM, FRCP, FRCPsych Manchester Royal Infirmary; Manchester, UK

1 Depression and antidepressants. Newcastle upon Tyne: Prescription Pricing Authority, 1995.

2 Livingston MG, Livingston HM. New antidepressants for old people? BMJ 1999;318:1640-1.

3 Thomson O'Brien MA, Oxman AD, Davis DA, et al. Educational outreach visits: effects on professional practice and health care outcomes. Cochrane Database Syst Rev 2001:(2):CD000409.

4 Henry JA. Epidemiology and relative toxicity of antidepressant drugs in overdose. Drug Saf 1997;16:374-90. 